Chicago was suffering with frightful pains in the head, optic neuritis, etc., that were attributed to a brain tumour or cerebral abscess and that an operation was contemplated. The family were advised by telegraph to have the nasal sinuses examined, and if an operation was performed, to open the sinuses before trephining. In reply to inquiries it was learned that the pain in the head had gradually increased in severity, and later a hæmorrhagic neuroretinitis and an involvement of the ocular muscles had appeared. The diagnosis was tumour cerebri or a cerebral abscess. There was difficulty in determining the location of the lesion, and unless the patient showed some improvement in a day or two a craniectomy would be performed; "we will see the case with Dr. Fish, but we know that if he saw the patient he would not think it was a sinus case." This case, under large doses of K. I., went on to perfect recovery without operation. The patient was examined in December last: the eyes were normal ; the nostrils congested; the turbinates, both lower and middle, swollen; no attempt was made to examine the sinuses and no astringents were applied. In this case syphilis, hereditary or acquired, is not to be thought of. As to the diagnosis in this case, was it a cerebral abscess that was absorbed, a brain tumour that was arrested, or sinus disease (capable of causing all the symptoms) that healed spontaneously, as it often does?

(To be continued.)

\title{
THE \\ TREATMENT OF FOREIGN BODIES IN THE RESPIRATORY TRACT AND CESOPHAGUS.
}

An Address delivered before the American Laryngological, Rhinological, and Otological Society, at the Thirteenth Annual Meeting, held at New York City, May 30, 1907.

\section{By Professor Gustav Killian, Freiburg, in Breisgau.}

The following is an abstract of an address delivered before the American Laryngological, Rhinological, and Otological Society at the Thirteenth Annual Meeting held at New York City, May 30, 1907. Speaking of foreign bodies in the respiratory tract, Professor Killian said:

I need not say much with reference to the upper respiratory tract. We are often called upon to treat foreign bodies in the nasal cavities, especially in children. As a rule, they are buttons, 
beads, etc., made out of various materials, cherry-seeds, beans, peas, and occasionally a forgotten cotton tampon. Nasal calculi also belong to this class.

Once I was called upon to remove a free osteoma, of the size of a hazel-nut, which had been left in the nasal cavity after an operation performed the year before, and which rolled to and fro with the movement of the head. It was incomprehensible how the patient could endure the condition so long. Another case was that of a child who accidentally ran a splinter into his nose. Another infant was brought to me suffering from very high fever, bad smelling nasal discharge, and swollen glands, resulting from a paper plug which his sister had stuck into his nose some time before. That the severe clinical picture depended upon this was shown by its disappearance after removal. Finally I must mention a case in which a wooden plug, as thick and long as the little finger, was shot through the maxillary sinus, passing into the nose and perforating the septum.

Foreign bodies in the nasal cavities in children are generally found in the vicinity of the vestibule, if no unskilful efforts at removal have been made. In one of my cases, the ethmoid bone was penetrated as far as the orbit in the fruitless search for the foreign body. Inflammation symptoms may follow such efforts, which may make the discovery of a foreign body very difficult.

The simplest and the most common method of removal is to introduce a thick nasal probe, bent forward, beyond the foreign body, and then draw it from behind forward and remove it. The little patient often, involuntarily, assists in this by moving the head backwards. A strong pair of toothed forceps is the best instrument to use for large impacted foreign bodies. When necessary, a great amount of strength may be used with this instrument, so that the foreign body may be extracted with certainty.

So far as foreign bodies in the accessory cavities of the nose are concerned, the maxillary sinus is the principal site. They are generally trocars and drainage tubes which are shoved into artificial openings. Occasionally, roots of teeth or portions of bone remaining after operation are found. I have often succeeded in locating and extracting a drainage tube through an alveolar fistula by means of a nasal speculum. I have occasionally removed such objects while performing the radical operation.

Foreign bodies are seldom found in the epipharynx. I was once called upon to remove a collar button which I had drawn out 
of the larynx, under narcosis, with the head hanging downwards; it slipped from me and fell into the epipharynx. Its removal was more difficult than I had anticipated.

In the mesopharynx and hypopharynx, fish-bones are most frequent. They are found in the region of the tonsils, sometimes at the base of the tongue, and rarely in deeper positions. They are readily removed with forceps. Pieces of bone may be lodged in the sinus pyriformis. I was called upon to remove a splinter of bone, sharp at both ends, which was stuck in this place, causing the patient to have severe symptoms, though it was not discovered by the physician in charge. In another case, deep pharyngeal and laryngeal abscesses, resulting from a chicken-bone sticking in the insus pyriformis, were spontaneously evacuated and cured.

Foreign bodies in the larynx constitute an extensive and interesting chapter of our subject, but are not so common as generally supposed. As a rule the foreign body immediately travels further down. Sharp and uneven objects are most apt to be caught in the larynx : needles, fish-bones, bone splinters, shells of nuts. Once I saw half of a dental plate lying there.

The laryngoscopic method of extraction has, in this particular, achieved a great triumph. It may be called the normal method for adults, as long as the foreign body is not firmly wedged in. In children, the removal through the agency of the laryngoscope is very difficult, and though it may be successful under narcosis, still our old time technic has its limitations. It entirely fails when the foreign body is tightly wedged in. The smaller the child, the greater the difficulty. It was formerly necessary to take refuge in laryngo-fissure, but now we have the additional advantage of direct laryngoscopy, which makes laryngo-fissure superfluous in the majority of cases. In all doubtful cases in children, I should recommend the practice of entering directly with the tube, under narcosis, and with the head hanging down.

The number of cases treated so far in this way is small (Denker, Garel, Guisez, De Stella, G. Killian), but it justifies the greatest hope for the future. I recommend you to use my tipped tube spatula. The base of the tongue is drawn forward with it and the epiglottis and inner surface of the larynx are cocainised. Under narcosis this is done with the head hanging down. The spatula is then passed over the epiglottis, which is thus drawn forward. By suitable illumination with an electric forehead lamp (Kirstein's), or with an electric extension lamp, a beautiful view into the larynx is obtained, not only as far as the vocal bands, but also 
into the subglottic space and deeper, provided the local and general anæsthesia is sufficient. Foreign bodies can be readily seen and removed with suitable forceps. If the process is made difficult by the mucus that collects, this is remored by my pump (which has lately been modified by my assistant, Brünings). Anyone who has had experience can, under certain conditions, avoid tracheotomy in recent cases, if the danger of suffocation is not too great, by rapid performance of direct laryngoscopy, as Denker succeeded in doing in one case.

\section{Foreign Bodies in the Trachea and Bronchi.}

I come now to the treatment of foreign bodies in the trachea and bronchi. In these, the direct method has the field entirely. The results have been extraordinarily successful. Since 1897, when I reported my first case in the Mïnchener med. Wochenschrift, until the end of March this year, 164 cases have been reported. (I count only the cases in which a foreign body was actually present.) To my certain knowledge, however, there are a large number of cases still unreported, so that altogether there are 200 or more. Practitioners have made themselves familiar with the new method in most civilised lands. It was very early and ardently employed in the United States.

In order to reach a foreign body in the trachea or bronchi, it is necessary to introduce a tube of proper calibre and proper length through the glottis, that is, to perform upper direct tracheobronchoscopy.

The tube should be chosen just wide enough to pass smoothly through the larynx. In adults, its calibre should be from 9 to $14 \mathrm{~mm}$., and in children, as a rule, it must be reduced to $7 \mathrm{~mm}$. Very small children require tubes of $5 \mathrm{~mm}$. or less in diameter. The length of the tube should correspond, in adults from 30 to $40 \mathrm{~cm}$, in children from 20 to $30 \mathrm{~cm}$., or even less. The actual measurement for the removal is to be taken from the upper row of teeth to the point where the foreign body lies, with the head bent back. This is easily ascertained before the operation.

The variations in the age of patients, the size of their bodies, the width of the larynges, and the location of the foreign bodies are so great that a great many different tubes must be kept on hand. Furthermore, it sometimes happens that a change from a longer or shorter tube is necessary during the operation. In order to secure a desirable simplicity in this particular I have effected 
the lengthening of the tube by introducing secondary tubes. The sliding tubes of Brünings, which I now show, have been constructed accordingly, and are very efficient for this purpose. They are composed essentially of a tube spatula, through which a second tube may be pushed as far as desired. An equipment of five or six of these tubes is sufficient for all adult cases. A regulator attached to the lateral wall of the tube spatula controls the introduction of the tube. The tube spatula is readily inserted and passed through the glottis. We can easily introduce the sliding tube any distance from the middle of the trachea to the bronchi of the lower lobes. Two lateral openings in the sliding tube renders respiration easier. They are especially necessary when it is desired to introduce a tube, filling the larynx, into a branch bronchus, where the lung tissue is not sufficient for the purpose of respiration (shut off by foreign body or tissue changes).

Good local and general anæsthesia must be secured in order to introduce such a tube into the deeper air passages. A hypodermic of $\cdot 01$ to $\cdot 02 \mathrm{grm}$. of morphine is given to adults a half hour before; in children, the internal administration of codeine, 5 to 15 drops of a 1 per cent. solution, according to age, will be advantageous.

When no narcosis is to be employed, the larynx and trachea are cocainised by the aid of a mirror while the patient is in the sitting position. In this connection, the epiglottis may be pulled so far forward that a cocaine applicator, extended perpendicularly, introduced through the mouth, with the aid of a mirror, will glide over the posterior wall of the larynx into the deeper parts. This procedure may also be undertaken by direct laryngoscopy or by the aid of the finger. Wherever I use general narcosis I cocainise only after it is sufficiently deep. Then the mouth speculum is introduced, the tongue seized with the forceps and pulled forward, and cocainisation is made by means of the tipped tube spatula, as before stated.

It is best, in performing direct bronchoscopy for the removal of foreign bodies, to have the patient in the recumbent position, whether or not a general anæsthetic is used. In this way all the disturbances, so easily called forth by the accumulated saliva and mucus, are suppressed.

The patient should be placed so that the foreign bodies lie at the highest level and the head at the lowest, since in this way the clearest field of view is obtained. Generally I have the patient lie on his back, but lately I have begun to place him on his side, which seems to be the most advantageous position. 
It is a great mistake to bend the head too far back at the introduction of the tube spatula. One should begin with a moderate bending of the head backwards and increase this during the procedure as far as occasion demands. The tube spatula is introduced through the angle of the mouth from the opposite side, if the situation of the foreign body is known. The epiglottis, arytænoids, and vocal bands are sought and the instrument is then passed between them into the trachea. If the cocainisation has been sufficient, there will be no interference at the glottis.

After the deep portions and the bronchus especially have been carefully cocainised (without pushing the foreign body deeper), the sliding tube may be introduced first to the bifurcation and then into the affected bronchus. It should be advanced until it reaches the neighbourhood of the foreign body, which is often covered with mucus and granulations or but slightly visible. It is then necessary to suck up the secretion with the pump. The granulations must be carefully treated so that no hæmorrhage from in front occurs.

If the foreign body does not lie in a position where it may be easily removed, the neighbouring mucous membrane should be made anæsthetic by the application of cocaine on a small cotton carrier or by a cocaine spray (weak solution), inasmuch as this region is hyperæsthetic. The slightest movement of the foreign body causes severe paroxysms of coughing. The greatest difficulty results from neglecting to cocainise in this way, for it is clear that everything else has been made anæsthetic, especially the whole passage-way to the foreign body, except the mucous membrane directly adjoining.

The procedure is much simpler in tracheotomised patients, whether tracheotomy has already been performed or whether we ourselves have been forced to perform it on account of the severe dyspnœa, or must perform it because the extraction cannot be accomplished by the upper method.

Lower direct tracheo-bronchoscopy seldom requires general anæsthesia. Simple cocainisation of the tracheo-bronchial mucosa is sufficient. Larger and shorter tubes may be used. For tho reason already stated, it is best to have the patient in the recumbent position. As a rule, Brünings' new instruments are the best adapted for lower tracheo-bronchoscopy.

He divided the foreign bodies into two groups, namely those which were hard, such as fish-bones, nails, steel pens, coins, pebbles, prune-stones, beads, cherry-stones, coffee-beans, pieces of 
bone, teeth, metallic objects, shirt and collar buttons, artificial teeth. Having dealt with the methods adopted in such cases he described the means of extracting hollow bodies, such as fragments of cannulæ pencils, whistles, pen-holders; those bodies are breathed into the bronchi and can be reached by upper bronchoscopy and grasped at the edge with forceps. If the edge of the foreign body is covered with swollen bronchial mucosa, it is better to use Killian's own body forceps, which, after being pushed in the opening, spread their branches in the hollow body and give a reliable hold.

Speaking of bodies which are not hard, such as melon-seeds, oranges, dates, cereal spikes, expansible fruit kernels, soft beans, Killian says, as far as the technique is concerned upper and lower tracheo-bronchoscopy may be used to remove the foreign bodies. Extraction forceps are satisfactory. Bodies that break easily, such as nut kernels, must be removed with great care, because if broken with the forceps a number of foreign bodies are made out of the one, and complications may result, especially with small children. He mentioned many cases involving soft and coherent bodies, such as cotton, pieces of meat, quill of an arrow, soft down, bodies that were soft but not coherent such as pieces of turnip, plant leaves, pieces of fruit, and other things as of serious moment if of large size. Those bodies that are not coherent should be removed with bean-forceps.

The address also dealt with the removal of foreign bodies from the osophagus, and Killian mentioned that he had had seventeen cases of this class, all of which were promptly cured by his method of removal.

\section{Discussion.}

Dr. Chevalier $J_{A C K s o n}$, of Pittsburg, Pa., expressed the obligation that all laryngologists are under to Professor Killian for opening a new field, one already fruitful and large with promise. That bronchoscopic work was done at all, and that it was done safely and effectively, was due to him. In his own work Dr. Jackson had had the utmost satisfaction with the tube designed after Dr. Killian's original pattern, which he believed would never be improved in any essential particular. The method of illumination, however, presented some difficulties. Bronchoscopy with the Kirstein or any other head-light was practical in many but not in all hands. It demanded not only a natural endowment of dexterity, but also such clinical opportunities of practice as come to but few. He had, therefore, added to the Killian bronchoscope a light carrier in a separate tube, and this modification, he believed, rendered the work easier. It could not be hoped to go beyond Professor Killian's own results, but the light carrier at the end of the tube would extend the 
usefulness of his method by making the instrument effective in the hands of men of lesser skill. Bronchoscopic work for the present concerned itself mostly with foreign bodies, and it was highly desirable that cases should be dealt with by some one immediately at hand. In every centre of population there should be at least one man competent to explore the bronchi. Every effort should be made, therefore, to devise easier means of work. With the tube which he exhibited any man accustomed to the direct inspection of the larynx might, without hesitation, explore the tracheo-bronchial tree to its second and third subdivisions. In no case of foreign body or exploration for disease was damage done. In no case was shock apparent. The dangers of superior bronchoscopy were those of general anæsthesia. The dangers of lower bronchoscopy were even less. His preference was for upper bronchoscopy, as . with distally illuminated tubes and the accessory drainage canal in the wall of the tube, the view was as good in a long tube as in a short one. Since the development of Professor Killian's work he could conceive of no foreign body case suitable for expectant treatment. What may be done with the bronchial tube in diseased conditions was a question for the future to determine, but the prospect was bright. He believed that in the near future practically all endo-laryngeal operations will be done by the direct method, and that many of the more important examinations will be made in the same way. With the patient in proper position, the larynx thoroughly cocainised, the passage of the tube down to the tracheal bifurcation was so simple a proceeding that it might well be made a matter of routine. A few years ago the diagnosis of simple local lesions below the larynx was very imperfect and the treatment was almost impracticable, but Professor Killian's work had shown how to deal with these conditions. Upper bronchoscopy had a considerable field of usefulness as a routine method as an adjunct to the direct examination of the larynx. Where the indication was primarily of tracheal or bronchial trouble, and particularly where operative work was indicated, lower bronchoscopy was a somewhat easier operation. With the tracheotomy done under infiltration anæsthesia, he believed it often to be safer. With a separable speculum there was not the slightest difficulty in promptly passing the bronchoscope through the glottis. When he first used Professor Killian's separable spatula he considered it a device second in importance only to the bronchoscope itself. In a modified form he had found it so useful that he was loth to abandon it.

Dr. E. Fletcher Ingals, of Chicago, wished to add his expression of gratitude to Professor Killian for developing bronchoscopy. There was, however, much to be done to ensure the safety of the operation. He had not used Professor Killian's pump for removing mucus from the bronchi, but had adapted Jackson's pump for the ear to this purpose. He emphasised the importance of pulling out the tongue. One must learn from experience the effect of the drugs that may be applied to the mucous membranes for the purpose of preventing bleeding, and how much may be used with safety. There was considerable danger from the use of too much of either cocaine or adrenalin. His preference was for upper bronchoscopy, which he would try first, particularly in girls and women, for a reasonable time--not more than fifteen minutes-ind if not successful would then do tracheotomy. He had had no success at all with the electric magnet. With a pin-finder, something like a corkscrew, he had been able to get pins into the middle of the lumen of the bronchus; then to shove the bronchoscope down over them and remove them with forceps. There was danger from the little hooklets recommended in Pro- 
fessor Killian's earlier paper, as they might easily become caught in a small bronchus and could not be removed except by tearing out, which would be likely to result in emphysema and death. The swelling of the larynx after bronchoscopy had sometimes required speedy tracheotomy. $\mathrm{He}$ cited a case in which he had removed a foreign body by upper bronchoscopy. The following morning the child seemed to be suffocating from swelling of the larynx; he introduced a Dwyer's tube, which was later coughed up, and there was no further trouble. He had seen three cases die after what he would consider easy operations, in which he was sure there had been no perceptible injury to the air-passages. The chief questions which it seemed important to consider were, What causes the danger? How long the operation may be continued? and, What symptoms indicate that the operation is being carried too far?

Dr. A. Coolidge, of Boston, Mass., said that a few years ago if a foreign body were inhaled, it was either let alone in the hope that it would be coughed up, or tracheotomy was done, and forceps were blindly pushed into the trachea and bronchi, in the hope of seizing it. The mortality of these two methods had not differed enough from each other to establish a principle of action. The first great step in advance was the demonstration that a straight hollow tube could be inserted through a tracheal wound, and the lower trachea and primary bronchi seen by direct inspection. By this simple proceeding, with a comparatively simple armamentarium, every surgeon might be ready to successfully reach a large proportion of inhaled objects. Foreign bodies presented a large variety in size and shape, and consequently varied much in the ease with which they might be removed Every help in the avoidance of injury, time, and the danger of infection, might in any case mean the difference between life and death. Consequently a carefully devised series of instruments, and a well-studied technique, were necessary for the greatest success. For this advance in medical efficiency, the medical profession and the patient were indebted to Professor Killian. It would be a long time before even a majority of medical men could find within an available distance, when the case arises, either the instruments or the skill to extract a foreign body in the way in which it would be done in Professor Killian's clinique. For sereral reasons the lower route would be the safer one for a limited equipment, and the question of choice between upper and lower bronchoscopy should properly depend fully as much upon the available equipment both of instruments and skill as upon other conditions. It should always be borne in mind that if a tracheotomy will probably be necessary, prolonged attempts by the upper route might exhaust the patient more than is warranted. The important thing with reference to light is that there should be enough. Until the relative value of different forms of light becomes definitely fixed and the apparatus therefore universally available, there could not be too many resources, if ther are efficient. If enough light could be forced into the tube from above without interfering with the work, that was all that was necessary. Practically, however, many in this country had found that the small distal lamp gives so bright a light, and is maintained by so simple and portable an electric supply, that it was likely to be retained for the present, at least, as a substitute. He had not found that secretion shuts off the light any sooner than it would shut off the view of the field if the light came from above. It did add a complication to the tube, and was a source of possible danger by breaking. The light was also more uneven and did not penetrate so far beyond the end of the tube as a powerful light from above. He reiterated what Professor Killian had said, that 
to do good work and to avoid injury the instruments must be fitted to the particular case, which meant anticipation beforehand of all possible conditions.

Dr. Frank B. Sprague, of Providence, R. I., called attention to the use of atropine for the purpose of fortifying respiration and drying the secretions. He had employed it successfully in three cases.

Professor Killian, in closing the discussion, said that irritation of a foreign body in a bronchus would very probably cause bronchitis or pneumonia, in which event it was important to understand the general condition of the patient, and whether bronchoscopy should be performed. Some cases were so dangerous that the patient would die unless the foreign body was removed; in such cases the method should be continued and the child be given the benefit of possible relief.

\section{gostracts.}

\section{MOUTH.}

Roy, J. N. (Montreal).-Primary Melanosis of the Palate; Naso-buccal Fistula of Recent Sarcomatous Origin. "Montreal Medical Journal," November, 1907.

This is an exceedingly interesting case on account of its rarity, the writer having found only two similar cases on record. The patient, a blacksmith, when twenty-three years old, injured his palate slightly with the stem of a clay pipe. One year later he discovered in the medium raphe of the vault a small round spot 3 millimetres in diameter. During the following twelve years this spot increased in diameter to about 6 millimetres. The only symptom was slight roughness of the tongue on pressure. About this time iodine was applied, and pain commenced to appear, which lessened when it was discontinued. The extension of the disease, however, was continuous, pigmentation taking place in the surrounding parts as well as the original site. Four years later all the space within the dental arch of the superior maxilla was filled with melanotic granulations. At the end of another four years, while the general granulation had markedly increased, there was depression of the palate on the left side, due to the formation of a naso-buccal fistula. This was attended by neither hæmorrhage nor suppuration.

Examination now revealed melanosis of the entire hard palate. Granulations of a brown or blackish colour were scattered all over, the left side being greatly depressed. At a point between the middle and posterior thirds of the hard palate a probe passed readily into the nasal cavity.

There was no dysphagia, but the voice was nasal, and the pharyngeal reflexes were absent.

Microscopic examination led to a diagnosis of "melanotic sarcoma resembling melanotic endothelioma."

As the patient positively refused operative measures, the necessary mutilation together with the possible complications and doubtful prognosis being explained to him, the case was allowed to progress, under resorcin and hygiene treatment, toward the inevitable fatal issue. 